diagnostic guidelines of European Society for Paediatric Gastroenterology, Hepatology and Nutrition (ESPGHAN).

Out of 1404 children $(51 \%$ female, mean age 7.23 y) tested, $85(6.05 \%)$ had positive rapid POC test. All those were referred to do serology testing but

2 were excluded from the analysis as were older than firstgraders and 4 refused. Out of 79 tested, 8 had positive IgA tTG (6 with values of more than 10x upper limit of normal $(\mathrm{ULN})$ ). Finally, 7 children were diagnosed with CD (0.5\%): one with no-biopsy approach according to ESPGHAN guidelines and 6 with positive biopsies (one had Marsh 2 and 5 Marsh 3). One child (tTG was 4x ULN) had normal biopsy and $\mathrm{CD}$ was not confirmed. There was no significant difference in children with $\mathrm{CD}$ and the children with negative serology in age (median 7.08 vs 7.25 years, $\mathrm{P}>0.05$ ), BMI (median 16.39 vs $15.36, \mathrm{p}>0.05$ ), presence of symptoms $(57 \%$ vs $44 \%, \mathrm{p}>0.05)$ nor in positive family history for CD (none vs one).

Children diagnosed with CD did not have significantly more symptoms nor lower BMI, and could not have otherwise been diagnosed clinically. In our study, the prevalence of CD in first-grade children was overall $0.5 \%$. This is higher prevalence of $\mathrm{CD}$ than reported earlier for Croatia, showing the benefit of IgA and IgG DGP based POC test in screening.

\section{COLECTOMY RATE IN PAEDIATRIC-ONSET ULCERATIVE COLITIS - ONE CENTRE EXPERIENCE}

Mario Mašić*, Ivana Trivić, Iva Hojsak, Oleg Jadrešin, Sanja Kolaček, Zrinjka Mišak. Children's Hospital Zagreb

10.1136/archdischild-2021-europaediatrics.240

Paediatric-onset ulcerative colitis (UC) is often more extensive than in adults, and as disease severity is associated with disease extent, children are more prone to refractory severe episodes, sometimes requiring colectomy.

Previous population-based studies in patients with UC revealed variable colectomy rates. However, a decrease in colectomy rates was observed during the last two decades. The aim of our study was to assess the colectomy rate in paediatric patients with UC and to compare the clinical features of children who had to those who did not have colectomy.

In our hospital, data on children diagnosed with inflammatory bowel disease have been prospectively collected since January 2004. Retrospectively we analysed data (disease history, age at diagnosis, sex, baseline characteristics, and course of disease) on all children diagnosed with UC $(n=170)$ from 2004 to January 2018. Four children were lost to follow-up and were not included into analysis.

Of 166 children diagnosed with UC, 12 had colectomy (7.2\%). Patients with colectomy, compared with UC patients who did not have colectomy, did not significantly differ in gender (girls $58 \%$ vs. $48 \%$ ), age at diagnosis (12.27 vs. 12.62 years), body mass index at the time of diagnosis (median -1.2 vs. -0.22 ), Paediatric Ulcerative Colitis Activity Index (PUCAI) at the diagnosis (median 32.5 vs. 40), proportion of patients with extensive disease (E4) (75\% vs. 57\%) nor in extraintestinal manifestations (for all $\mathrm{p}$ was $>0.05$ ). There was a significant difference in family history positive to IBD $(25 \%$ vs. $7 \%, \mathrm{p}<0.05)$, in the highest PUCAI each patient had during the observation period (65 vs. 40, p < 0.05), number of patients treated with azathioprine $(92 \%$ vs. $28 \%$, p < $0.01)$ and anti-TNF therapy $(58 \%$ vs. $3.9 \%, \mathrm{p}<0.01)$. We found a significant decrease of colectomy rate (from 2004 to 2010 rate was $13.5 \%(7 / 52)$ vs. $4.4 \%(5 / 114)$ in the period from 2011 to 2018, p < 0.05). During the same time, the proportion of children with UC treated with anti-TNF therapy increased, although not significantly $(\mathrm{p}>0.05)$ : from 2004 to 2010, two children $(3.8 \%)$ were treated with anti-TNF therapy, and both required colectomy, while in later period, 11 children $(9.7 \%)$ received biologics, and 5 of them underwent colectomy.

Our paediatric patients presented with extensive form of the ulcerative colitis in nearly $60 \%$ of patients, requiring colectomy in overall $7.2 \%$ of patients. However, the colectomy rate decreased significantly during the observed period.

\section{ACUTE LIVER FAILURE IN NEWLY DIAGNOSED WILSON'S DISEASE - RECOVERY WITHOUT TRANSPLANTATION}

Mario Mašić*, Ivana Trivić, Ana Močić Pavić, Iva Hojsak, Sanja Kolaček, Zrinjka Mišak, Alenka Gagro, Ivančica Škarić, Tonći Grmoja, Martin Ćuk, Jurica Vuković, Oleg Jadrešin. Children's Hospital Zagreb

\subsection{6/archdischild-2021-europaediatrics.241}

Wilson's disease (WD) is an autosomal recessive genetic disorder that leads to the impairment of cellular copper metabolism.

Clinical presentation is heterogeneous, with predominantly hepatic, neurological and psychiatric manifestations. Acute decompensated WD presenting as fulminant liver failure is a life-threatening condition for which liver transplantation is the ultimate treatment.

14-year-old girl presented with acute abdominal pain and peripheral oedema lasting two weeks before onset of abdominal pain. On initial examination, patient was febrile, complaining of periumbilical pain, dyspnea, cough, presenting with anasarca, extensive limb oedema and ascites (gained $17 \mathrm{~kg}$ ), without encephalopathy. Laboratory evaluation revealed Coombs-negative haemolytic anaemia (Hb 101 g/L, Rtc $125 \times 109 / \mathrm{L})$, thrombocytopenia (79x109/L), leukocytosis $(19.27 \times 109 / \mathrm{L})$, mildly elevated inflammatory markers, hypergammaglobulinemia (IgG $21.7 \mathrm{~g} / \mathrm{L})$ with reduced complement components $\quad(\mathrm{C} 3 \quad 0.24 \mathrm{~g} / \mathrm{L}, \quad \mathrm{C} 4<0.08 \mathrm{~g} / \mathrm{L}), \quad$ coagulopathy (INR $>2.5$ ), marked hypoalbuminemia (>15 g/L), mildly elevated bilirubin $(49 \mu \mathrm{mol} / \mathrm{L})$, slightly elevated liver enzymes (AST $63 \mathrm{U} / \mathrm{L}$, GGT $98 \mathrm{U} / \mathrm{L})$, borderline serum ammonia (52.3 $\mu \mathrm{mol} / \mathrm{L})$, normal ALT and alkaline phosphatase. Abdominal ultrasound showed large amounts of ascites, with mildly enlarged, structurally altered liver (irregular surface, nodular parenchyma) and splenomegaly, with no signs of hepatic vein thrombosis on Color Doppler and MRI. Heart ultrasound was normal. Kayser-Fleischer ring was negative on slit lamp examination. Viral serology (HAV, HBV, HCV, HEV, EBV, CMV, and Parvo B19) was negative as well as autoantibodies (ANA, SMA, LKM, ANCA, anti dsDNA). Serum alpha-1 antitrypsin level was normal. Diagnosis of Wilson's disease was practically confirmed after demonstrating pronounced cupriuria (17.8 $\mu \mathrm{mol} / 24 \mathrm{~h})$; serum ceruloplasmin was low $(0.13 \mathrm{~g} / \mathrm{L})$ and serum copper levels slightly reduced (10.1 umol/L). Initial treatment was supportive (albumin infusions, vitamin $\mathrm{K}$, fresh frozen plasma, diuretics, lactulose, antibiotics). Liver transplant 
was considered (on admission MELD score 24, King's Wilson index 12; on day 10 MELD score 18, King's Wilson index $8)$.

The decision was made to attempt plasma exchange over three days and introduce penicillamine. The patient improved clinically, tolerated penicillamine well and was released subsequently after 34 days of hospitalization and five plasma exchanges overall. Liver synthetic function completely normalized few months later. Genetic analysis of ATP7B gene (H1069Q mutation) confirmed presence of the H1069Q mutation on one allele.

We presented a patient suffering from WD in whom the disease presented acutely with development of liver failure with near-normal values of liver enzymes, mild hyperbilirubinemia, markedly impaired synthetic liver function and Coombs-negative haemolytic anaemia. Acute liver failure in WD may be successfully managed with plasma exchange therapy.

\section{ACUTE BILIARY PANCREATITIS OCCURRING IN THE EARLY POSTPARTUM PERIOD IN A TEENAGE GIRL: A CASE REPORT}

Mario Mašić*, Iva Hojsak, Oleg Jadrešin, Marko Mesić, Sanja Kolaček, Zrinjka Mišak. Children's Hospital Zagreb

\subsection{6/archdischild-2021-europaediatrics.242}

Introduction Acute biliary pancreatitis during early postpartum period in adolescent pregnancy is a rare, but challenging clinical entity in terms of diagnosis and management. We report a case of acute pancreatitis, occurring in early postpartum period in an adolescent patient who had an otherwise uneventful course of pregnancy.

Case Report A 15-year-old patient presented with a complaint of severe upper abdominal pain with propagation to the back and bilious vomiting, 18 days after delivery of a healthy child. Apart from psychiatric treatment due to behavioral disorder, she was healthy, and the pregnancy was uneventful.

The patient was initially examined and admitted to a secondary institution where the diagnosis of acute pancreatitis was established, due to clinical features, elevated amylase values and abdominal ultrasound findings. After primary stabilization, she was transferred to the Intensive Care Unit of our institution. When admitted, the patient was acutely ill, pale, and afebrile, with slow peristalsis and diffuse abdominal pain. Laboratory values of inflammation markers were elevated (CRP $135.4 \mathrm{mg} / \mathrm{l}$, PCT $1.29 \mathrm{ng} / \mathrm{mL}$ ), with significant increase in lipase (2602 U/L) and amylase in serum (666 U/L) and urine (13593 U/L). Abdominal ultrasound showed enlarged, intumescent pancreas, without necrosis or other focal changes, and multiple concrements in gallbladder, without intrahepatic or extrahepatic duct dilatation, the latter confirmed with magnetic resonance cholangiopancreatography. During hospitalization, patient's condition worsened with the development of a pseudocyst $(66 \mathrm{~mm}$ in diameter) in the tail of pancreas, free fluid in the abdomen and extensive bilateral pleural effusions, which was treated with antibiotics (meropenem), octreotide for the first three days and parenteral fluid, electrolyte and nutrition supplementation. After stabilization, the patient was admitted to Department of Gastroenterology. On the 14th day, a severe abdominal pain reoccurred with propagation to the back and vomiting, with an increase in inflammatory markers and amylase and lipase values.

Abdominal ultrasound showed active pancreatitis with necrotic focal changes in the pancreas. The aforementioned deterioration was inflicted by patient's self-initiated and covert ingestion of nuts and some snacks during the night. After stabilization, laparoscopic cholecystectomy was performed and the patient was discharged.

Conclusion Acute pancreatitis must be kept in mind when evaluating patients presenting with abdominal pain after child delivery, with gallstones being the most common etiological factor. Pregnancy and postpartum associated acute biliary pancreatitis usually has a mild-to-moderate clinical course with a favorable outcome, but can present a challenging clinical entity in adolescence, even posing a survival threat.

\section{RESEARCH OF NUTRITIONAL STATUS OF SURGICAL PATIENTS}

Indira I Khusainova, Anna N Zavyalova, Aleksandr Gostimsky, Oleg Lisovsky, Ivan Lisitsa, Valeriya P Novikova*. Federal State budgetary Educational Institution of Higher Education 'St. Petersburg State Pediatric Medical University' of tre Ministry of Healthcare of the Russian Federation

\subsection{6/archdischild-2021-europaediatrics.243}

Relevance Nutritional status (NS) has an effect on adaptation, pathological process severity, recovery rate, therapy effectiveness, hospitalization duration, etc.

Objective Evaluate NS of children being treated in clinical departments.

Materials and Methods nutritional status of 20 children 7 to 17 years old (average age is 13.8 years old) being treated in the surgical department was evaluated. Body weight, height and body mass index (BMI) as well as chest, waist, hips, hip and non-working arm wrist circumferences were studied; centile corridors (CC) were determined. All children have been evaluated for nutritional status using impedancemetry (body composition). All children were questioned using the nutritional status scale created specifically for this research. Statistical analysis, including parametric statistics methods, as well as Spearman's rank correlations was performed using the Statistics 6.1 software.

Results Children with chronic orthopedic pathology requiring repeated surgical intervention as well as children with acute trauma observed in the 2nd surgical department. Nutritional status was estimated according to anthropometric data using relative values (centile corridors). Height: $20 \%$ are tall (4 children in the 6th and the 7 th centile corridors), 30\% (6

children) are in the 3rd centile corridor, and 25\% (5 children) have stunted growth (the 1 st and the 2 nd centile corridors). Weight: $25 \%$ have excess weight (mainly children with acute pathology), 30\% (6 children) have moderate weight deficit (the 3 rd centile corridor), and 15\% (3 children) have acute weight deficit relative to height. Body mass index: $30 \%$ have excess weight and 20\% have acute deficit in nutritional status. Nutritional status was estimated by the method of impedancemetry, which coincided with the data of anthropometry. High reliable $(p<0.05)$ positive correlations between percentage of fat mass, lean mass, active cell mass and total water and the following anthropometric data: centile corridors of body mass and centile corridors of BMI were established.

Conclusions Thus, according to the totality of studies performed, $30 \%$ of children were in normal nutritional status; 